\title{
Hsa-miR-495 acts as a tumor suppressor gene in glioma via the negative regulation of MYB
}

\author{
BENPING ZHANG ${ }^{1}$, FEI YUAN ${ }^{2}$, JIE LIU ${ }^{2}$, YANG LI $^{2}$, FUCHENG ZHOU $^{2}$, \\ XUANXI LIU ${ }^{2}$, ZHEN HAO ${ }^{2}$, QINGSONG LI ${ }^{2}$, YONGRI ZHENG ${ }^{2}$ and WEIZHI WANG ${ }^{1}$ \\ Departments of ${ }^{1}$ Neurology and ${ }^{2}$ Neurosurgery, The Second Affiliated Hospital, \\ Harbin Medical University, Harbin, Heilongjiang 150086, P.R. China
}

Received June 12, 2015; Accepted April 18, 2016

DOI: $10.3892 / \mathrm{mmr} .2016 .5327$

\begin{abstract}
MicroRNAs (miRNAs) are small non-coding RNA molecules that regulate gene expression at the post-transcriptional level. Previous studies have reported that there are causative links between the abnormal regulation of miRNAs and cancer development. Hsa-miR-495 has previously been demonstrated to be downregulated, and to function as a tumor suppressor, in numerous types of human cancer. However, the function and molecular mechanism of hsa-miR-495 in glioma remains unclear. In the current study, the expression and effects of hsa-miR-495 on glioma were evaluated. It was identified that the expression levels of hsa-miR-495 were downregulated in glioma tissues and cell lines. Furthermore, restoration of hsa-miR-495 inhibited glioma cell proliferation and invasion in vitro. Notably, a luciferase reporter assay revealed that hsa-miR-495 was able to directly target v-myb avian myeloblastosis viral oncogene homolog (MYB) in glioma cells. In addition, an RNA interference assay indicated that MYB knockdown inhibited glioma cell proliferation and invasion in vitro. In conclusion, the results of the present study suggested that hsa-miR-495 may act as a tumor suppressor gene in glioma by directly inhibiting MYB expression, which may provide a novel therapeutic strategy for the treatment of glioma.
\end{abstract}

Correspondence to: Professor Weizhi Wang, Department of Neurology, The Second Affiliated Hospital, Harbin Medical University, 246 Xuefu Road, Harbin, Heilongjiang 150086, P.R. China

E-mail: wwz200300908@163.com

Professor Qingsong Li, Department of Neurosurgery, The Second Affiliated Hospital, Harbin Medical University, 246 Xuefu Road, Harbin, Heilongjiang 150086, P.R. China

E-mail: liqingsong0107@126.com

Key words: hsa-miR-495, glioma, suppressor, MYB

\section{Introduction}

Human gliomas, which originate from neural mesenchymal stem cells, currently account for $40-50 \%$ of nervous system tumors (1). In 2007, the World Health Organization classified astrocytomas into the following grades: Grade I-II, well-differentiated low-grade diffuse astrocytoma; grade III, anaplastic astrocytoma; and grade IV, glioblastoma multiforme (2). Despite the use of aggressive surgery in combination with radiotherapy, chemotherapy and biological therapy, gliomas remain difficult to treat, and the median survival of patients with glioma is 12-15 months (3). Therefore, it is necessary to elucidate mechanisms underlying the development of glioma and to discover novel targets for the treatment of glioma.

MicroRNAs (miRNAs) are a class of small non-coding RNAs that function as negative regulators of gene expression. miRNAs regulate expression at the post-transcriptional level by binding to complimentary sequences in the 3 -untranslated regions (3'-UTRs) of mRNAs $(4,5)$. Aberrant miRNA expression, and loss of the dynamic balance between oncogene and tumor suppressor gene expression, may lead to the formation and development of tumors (6).

Hsa-miR-495 is located at chromosome 14q32.31 in the human genome, the expression of which varies in different tumor types. Downregulation of hsa-miR-495 has been reported in numerous human malignancies, including non-small cell lung cancer, gastric cancer and acute myeloid leukemia (7-9). However, the function and molecular mechanisms of hsa-miR-495 in glioma remain unclear.

The present study aimed to explore the biological function and molecular mechanism of hsa-miR-495 in glioma. As hypothesized, hsa-miR-495 was downregulated in glioma tissues and cell lines. Notably, hsa-miR-495 was revealed to have vital roles in glioma cell proliferation and invasion by directly targeting v-myb avian myeloblastosis viral oncogene homolog (MYB), resulting in the inhibition of glioma cell invasion. These results indicated that upregulation of hsa-miR-495 may be considered a potential future therapeutic strategy for the treatment of glioma.

\section{Materials and methods}

Patient samples and cell lines. A total of 32 samples of paired human glioma and matched normal brain tissues were collected 
from adult patients that were admitted and diagnosed at The Second Affiliated Hospital, Harbin Medical University (Harbin, China). The 32 patients with glioma were comprised of 18 male patients and 14 female patients, including 12 low-grade glioma (5 grade I and 7 grade II) and 20 high-grade glioma (8 grade III and 12 grade IV). The age range of the patients was $45-78$ years old, with a median age of 62 . Tissue samples were immediately snap-frozen in liquid nitrogen and were stored at $-80^{\circ} \mathrm{C}$. Four human glioma cell lines (A172, U87, U251 and U373) and a normal neuronal primary human fetal glial (PHFG) cell line were purchased from the American Type Culture Collection (Manassas, VA, USA). The cells were maintained in Dulbecco's modified Eagle's medium (DMEM; Invitrogen; Thermo Fisher Scientific, Inc., Waltham, MA, USA) supplemented with $10 \%$ fetal bovine serum (FBS; Invitrogen; Thermo Fisher Scientific, Inc.) at $37^{\circ} \mathrm{C}$ in a humidified atmosphere containing $5 \% \mathrm{CO}_{2}$, and $1 \%$ antibiotics $(100 \mu / \mathrm{ml}$ penicillin and $100 \mathrm{mg} / \mathrm{ml}$ streptomycin sulfates) were added. The present study was approved by the Institutional Review Board of The Second Affiliated Hospital, and written informed consent was obtained from all patients.

RNA extraction and reverse transcription-quantitative polymerase chain reaction ( $R T-q P C R)$. Total RNA was extracted from the patient tissues and cell lines, subsequent to mincing and homogenization of the tissues, using TRIzol ${ }^{\circledR}$ reagent (Invitrogen; Thermo Fisher Scientific, Inc.) according to the manufacturer's protocol. Total RNA $(1 \mu \mathrm{g})$ was reverse transcribed using oligo (dT) 18 as a stem-loop RT primer (Qiagen $\mathrm{GmbH}$, Hilden, Germany) for the RT of miRNA, and using random primers (Invitrogen; Thermo Fisher Scientific, Inc.) for the RT of mRNA. The RT protocol was as follows: $60^{\circ} \mathrm{C}$ for $5 \mathrm{~min}, 4^{\circ} \mathrm{C}$ for $2 \mathrm{~min}, 50^{\circ} \mathrm{C}$ for $1 \mathrm{~h}, 85^{\circ} \mathrm{C}$ for $5 \mathrm{~min}$, and finally maintained at $4^{\circ} \mathrm{C}$. qPCR was performed using a Roche Lightcycler 480 Real-Time PCR system (Roche Diagnostics, Indianapolis, IN, USA). The total volume of the qPCR reaction was $20 \mu \mathrm{l}$, which contained cDNA template $1 \mu \mathrm{l}, 2 \mathrm{X}$ QuantiTect SYBR Green PCR Master Mix $(10 \mu \mathrm{l}), 10 \mathrm{X}$ reverse miScript SYBR Green PCR kit Universal Primer $(2 \mu \mathrm{l})$, forward primer $(0.5 \mu \mathrm{l})$ and RNase-free water $(6.5 \mu \mathrm{l})$. The qPCR cycling conditions were as follows: $95^{\circ} \mathrm{C}$ for $10 \mathrm{~min}$, followed by 45 cycles of $94^{\circ} \mathrm{C}$ for $20 \mathrm{sec}, 60^{\circ} \mathrm{C}$ for $30 \mathrm{sec}$ and $72^{\circ} \mathrm{C}$ for $30 \mathrm{sec}$. The specific primers for hsa-miR-495 and U6 were as follows: Hsa-miR-495, forward 5'-AGAAGTTGCCCATGTTAT-3', reverse miScript SYBR Green PCR kit Universal Primer (Qiagen GmbH); and U6, forward 5'-CTCGCTTCGGCAGCACA-3', reverse 5'-ACG CTTCACGAATTTGCGT-3'. The specific primers (Invitrogen; Thermo Fisher Scientific, Inc.) for MYB and glyceraldehyde 3-phosphate dehydrogenase (GAPDH) were as follows: MYB, forward 5'-TGACGAGGATGATGAGGACTTT-3', reverse 5'-ATCTGTTCGATTCGGGAGATAA-3'; and GAPDH, forward 5'-ACATCAAGAAGGTGGTGAAGCAGG-3', reverse 5'-CGTCAAAGGTGGAGGAGTGGGT-3'. All experiments were performed in triplicate. Relative expression levels were calculated according to the comparative $\mathrm{Cq}$ method (10).

Cell transfection. The hsa-miR-495 oligonucleotide and MYB-specific small interfering (si)RNA molecules were chemically synthesized by Shanghai GenePharma Co., Ltd. (Shanghai, China). The sequences were as follows: Hsa-miR-495 mimics, 5'-UGUGACGAAACA AACAUGGUGCACU-3'; and hsa-miR-495 mimics negative control, 5'-CAGUACUUU UGUGUAGUACAA-3'. Four MYB-specific siRNA sequences were synthesized, as follows: 5'-CGUUGGUCUGUUAUU GCCAAGCACU-3'; 5'-CAGAUGACUGGAAAGUUAUUG CCAA-3'; 5'-GGAAGGUUAUCUGC-AGGAGUCUUCA-3' and 5'-GCUUCCAGAAGAACAGUCAUUUGAU-3'. The following siRNA sequence was used as a siRNA negative control: 5'-UUCUCCGAACGUGUCACGUU-3'. Transfection was performed using Lipofectamine 2000 reagent (Invitrogen; Thermo Fisher Scientific, Inc.) according to the manufacturer's protocol. Cells were counted and seeded onto plates 1 day prior to transfection, in order to ensure suitable cell confluence. Hsa-miR-495 oligonucleotide and MYB-specific siRNA were used at final concentrations of $10 \mathrm{nmol} / \mathrm{l}$, and were transfected in antibiotic-free Opti-MEM medium (Invitrogen; Thermo Fisher Scientific, Inc.).

Cell proliferation assay. A172 and U87 cells were incubated in 10\% Cell Counting kit-8 (CCK-8; Dojindo Molecular Technologies, Inc., Kumamoto, Japan) diluted in normal culture medium at $37^{\circ} \mathrm{C}$, until visible color conversion was detected. Proliferation levels were determined 0, 24, 48 and $72 \mathrm{~h}$ post-transfection. CCK-8 solution $(10 \mu \mathrm{l})$ was added to each well, and absorbance was measured at $450 \mathrm{~nm}$ using a microplate reader (Model 550; Bio-Rad Laboratories, Inc., Hercules, CA, USA) after incubation for $2 \mathrm{~h}$ at $37^{\circ} \mathrm{C}$. All experiments were performed in triplicate.

Cell invasion assay. Cell invasion was determined using a Transwell assay. For the Transwell assay, the appropriate hsa-miR-495 oligonucleotide and MYB-specific siRNA molecules were transfected into A172 and U87 cells, according to the manufacturer's protocol. Subsequent to incubation for $48 \mathrm{~h}, 5 \times 10^{4}$ cells were seeded into the upper Matrigel-coated invasion chambers (BD Biosciences, San Jose, CA, USA) in serum-free DMEM. DMEM supplemented with $10 \%$ FBS was added to the lower chambers. After $24 \mathrm{~h}$, the invading cells were fixed using $95 \%$ ethanol and were stained with $0.1 \%$ crystal violet. A DM4000B microscope was used (Leica, Microsystems, Inc., Buffalo Grove, IL, USA). The assay was repeated in three independent experiments.

Bioinformatics. MiRanda (http://www.microrna.org) and miRcode (http://www.mircode.org) were used to predict the binding sites between hsa-miR-495 and MYB.

Dual luciferase assays. MYB mRNA 3'-UTRs containing the predicted hsa-miR-495 binding sites were amplified from human cDNA using PCR and were inserted into psiCHECK-2 luciferase reporter vectors (Promega Corporation, Madison WI, USA), in order to obtain constructed plasmids containing wild-type MYB mRNA 3'-UTR (psiCHECK-2-HMGB1-WT-3'-UTR). MYB mRNA 3'-UTRs containing mutation sequences in the putative binding site (psiCHECK-2-HMGB1-MUT-3'-UTR) were chemically synthesized by Shanghai GenePharma Co., Ltd. The MYB-WT or MYB-MUT recombinant construct plasmids and hsa-miR-495 mimics or negative control mimics were co-transfected into A172 and U87 cells using Lipofectamine 2000 (Invitrogen; Thermo Fisher Scientific, 
A

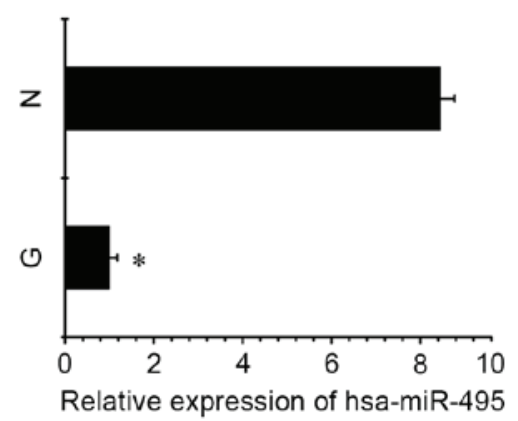

B

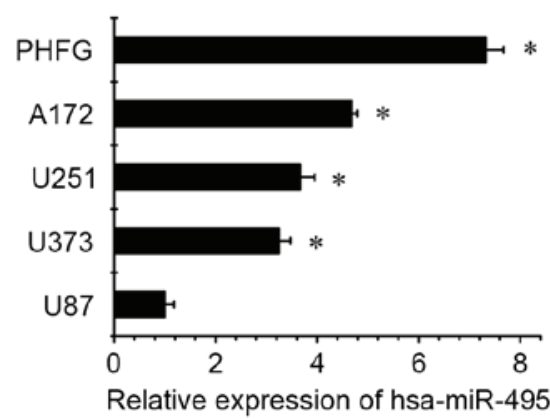

Figure 1. Hsa-miR-495 was downregulated in glioma tissues and cell lines. (A) qPCR analysis of hsa-miR-495 expression in glioma tissues and matched normal brain tissues. "P<0.005, vs. N. (B) qPCR analysis of hsa-miR-495 expression in normal neuronal cells (PHFG) and various glioma cell lines (A172, U251, U373 and U87). Data are presented as the mean \pm standard deviation $(n=3)$. Each experiment was performed in triplicate. ${ }^{*}<0.005$, vs. PHFG. N, matched normal brain tissues; G, glioma tissues; miR, microRNA; qPCR, quantitative polymerase chain reaction; PHFG, primary human fetal glial cells.

Inc.). Firefly and Renilla luciferase activities were measured $24 \mathrm{~h}$ post-transfection using the Dual Luciferase Assay (Promega Corporation) according to the manufacturer's protocol. All luciferase assays were carried out in triplicate. Luciferase activity was detected using the Modulus ${ }^{\mathrm{TM}}$ Single Tube Multimode Reader (Bio-Systems International, Beloit, WI, USA).

Western blot analysis. Cells were lysed in ice-cold cell lysis buffer [50 mM Tris, $\mathrm{pH} 8.0 ; 120 \mathrm{mM} \mathrm{NaCl} ; 0.5 \% \mathrm{NP}-40$; $50 \mathrm{mM} \mathrm{NaF}$; $1 \mathrm{mM}$ phenylmethylsulphonyl fluoride; $20 \mu \mathrm{M}$ sodium orthovanadate; $1 \mathrm{X}$ protease inhibitors (Invitrogen; Thermo Fisher Scientific, Inc.); $1 \mathrm{X}$ phosphatase inhibitors (Invitrogen; Thermo Fisher Scientific, Inc.)], and protein concentration was measured using a bicinchoninic acid protein assay kit (Pierce Biotechnology, Inc., Rockford, IL, USA). Proteins were separated by $12 \%$ sodium dodecyl sulfate-polyacrylamide gel electrophoresis and were electrotransferred onto polyvinylidene fluoride membranes (EMD Millipore, Billerica, MA, USA). The membranes were blocked with 5\% non-fat dried milk for $2 \mathrm{~h}$ at $37^{\circ} \mathrm{C}$, and were then incubated with mouse anti-MYB (1:500; ab17851; Abcam, Cambridge, UK) and mouse anti-GAPDH (1:5,000; ab8245; Abcam) antibodies overnight at $4^{\circ} \mathrm{C}$. Subsequently, the membranes were incubated with a horseradish peroxidase-labeled rabbit anti-mouse immunoglobulin G secondary antibody $(1: 10,000$; ab6728; Abcam) for $1 \mathrm{~h}$ at room temperature. Positive bands were detected using an enhanced chemiluminescence kit (Thermo Fisher Scientific, Inc.).

Statistical analysis. All experiments were repeated at least three times. Statistical analysis was performed using SPSS 17.0 (SPSS Inc., Chicago, IL, USA). Differences were assessed using Student's two-tailed t-test, while the CCK- 8 data were examined by analysis of variance. Data are presented as the mean \pm standard deviation. $\mathrm{P}<0.05$ was considered to indicate a statistically significant difference.

\section{Results}

Hsa-miR-495 is downregulated in glioma tissues and cell lines. In order to assess the expression levels of hsa-miR-495

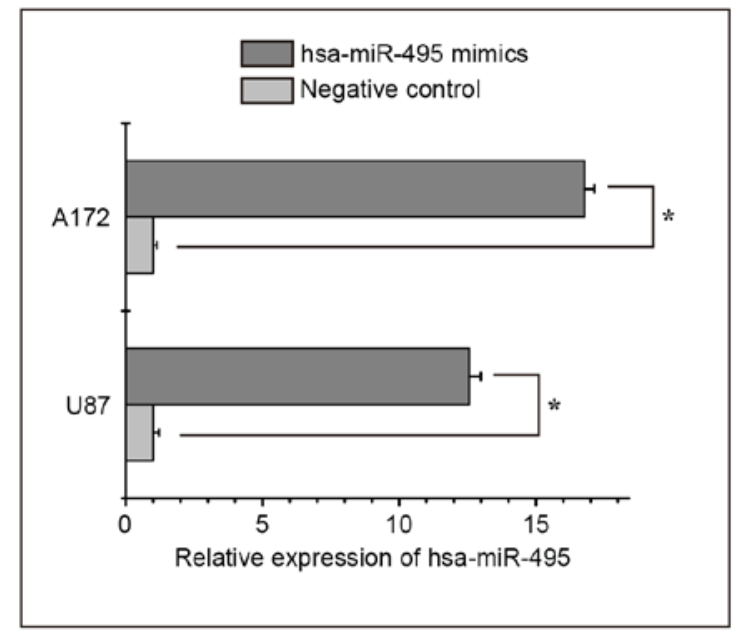

Figure 2. Restoration of hsa-miR-495 expression in A172 and U87 cells. Transfection efficiency of the hsa-miR-495 mimics and negative control mimics was detected in A172 and U87 cells by quantitative polymerase chain reaction. Data are presented as the mean \pm standard deviation $(n=3)$. The experiments were performed in duplicate and were repeated three times. ${ }^{*} \mathrm{P}<0.005$. miR, microRNA.

in glioma tissues, qPCR was performed on 32 pairs of glioma tissues and matched normal brain tissues. As presented in Fig. 1A, the expression levels of hsa-miR-495 were downregulated in glioma tissues compared with in the matched normal brain tissues. Furthermore, hsa-miR-495 expression levels were downregulated in the glioma cell lines (A172, U251, U373, and U87) compared with in the normal neuronal cell (PHFG) (Fig. 1B). These results indicate that hsa-miR-495 may have a role as a tumor suppressor gene in glioma. Each experiment was performed in triplicate.

Hsa-miR-495 inhibits glioma cell proliferation and invasion in vitro. Subsequently, the functional roles of hsa-miR-495 in A172 and U87 cells were explored. qPCR was performed to confirm that hsa-miR-495 expression was restored in A172 and U87 cells post-transfection with hsa-miR-495 mimics (Fig. 2). CCK-8 and Transwell assays indicated that increased expression levels of hsa-miR-495 markedly inhibited cell proliferation and invasion in A172 and U87 cells compared 

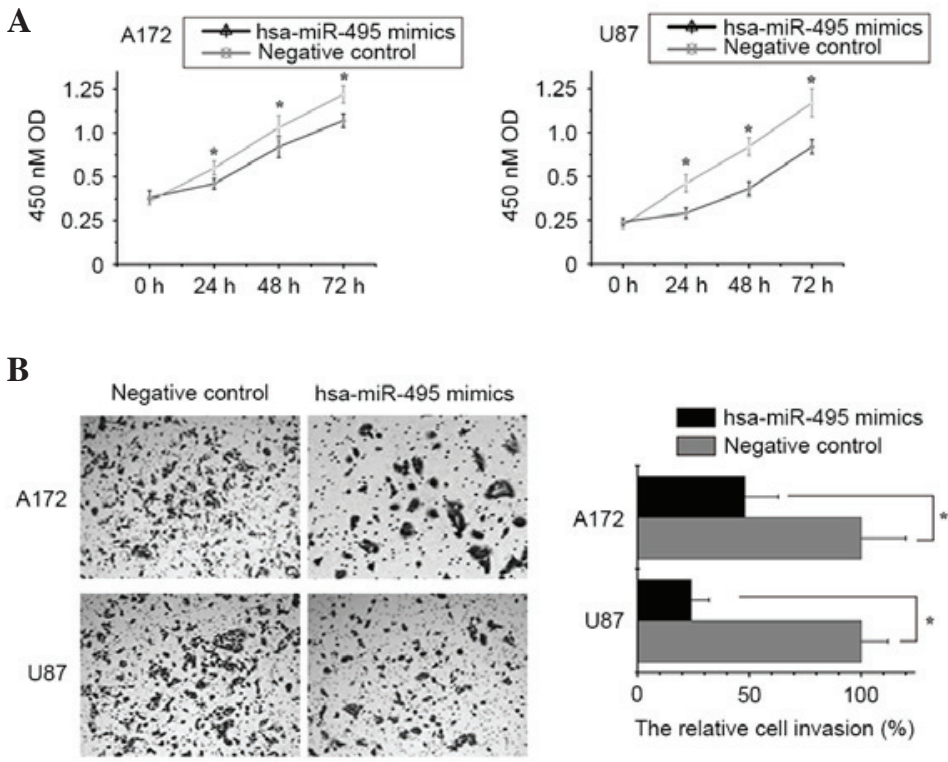

Figure 3. Hsa-miR-495 inhibits glioma cell proliferation and invasion in vitro. (A) Hsa-miR-495 mimics or negative control mimics-transfected A172 and U87 cells were subjected to Cell Counting kit-8 assays. (B) Hsa-miR-495 mimics or negative control mimics-transfected A172 and U87 cells were subjected to a Transwell invasion assay (magnification, $x 200)$. Data are presented as the mean \pm standard deviation $(n=3)$. All experiments were performed three times, and representative images are presented. ${ }^{*} \mathrm{P}<0.05$. miR, microRNA; OD, optical density.

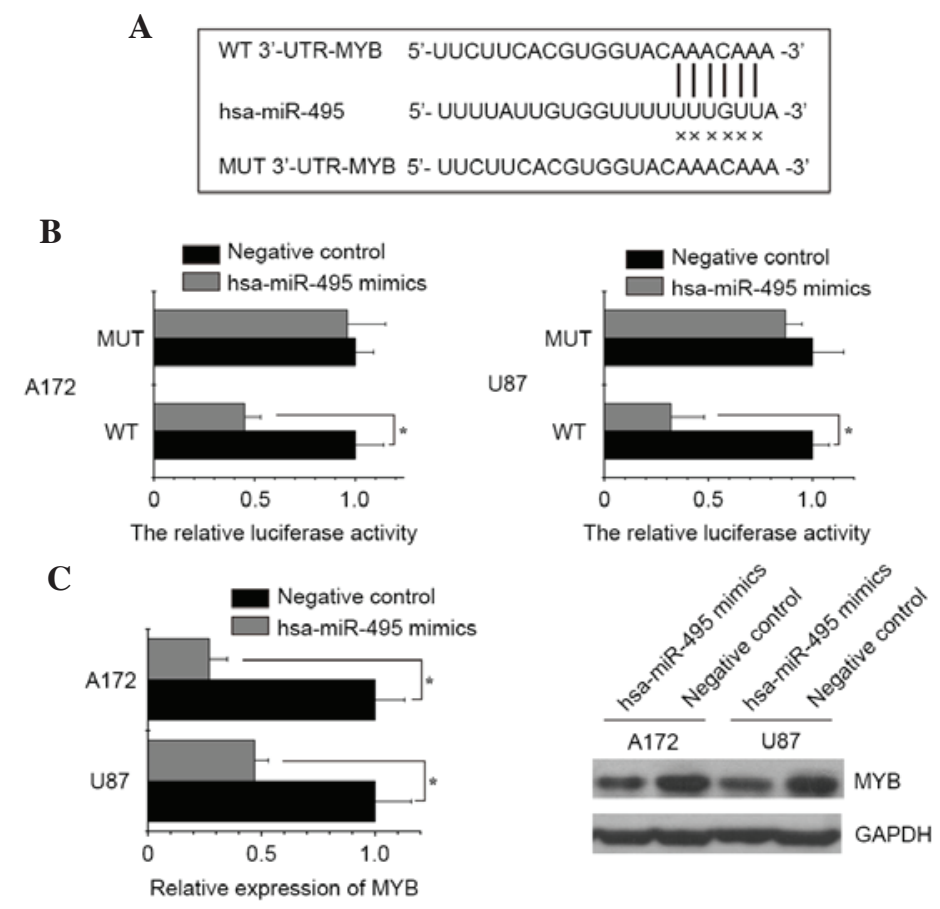

Figure 4. Hsa-miR-495 exerts its effects via negative regulation of MYB in glioma. (A) MYB mRNA 3'-UTR contains target binding sites for hsa-miR-495. (B) A luciferase activity assay was conducted on A172 and U87 cells, following co-transfection with WT or MUT 3'-UTR MYB constructs and hsa-miR-495 mimics or negative control mimics. (C) Quantitative polymerase chain reaction and western blotting were performed to determine MYB expression in A172 and U87 cells 48 h post-transfection with hsa-miR-495 mimics or negative control mimics. Data represent the fold change in expression, and are presented as the mean \pm standard deviation of three replicates. miR, microRNA; MYB, v-myb avian myeloblastosis viral oncogene homolog; WT, wild type; MUT, mutant; UTR, untranslated region; GAPDH, glyceraldehyde 3-phosphate dehydrogenase. ${ }^{*} \mathrm{P}<0.005$.

with in the control group (Fig. 3). These results suggest that hsa-miR-495 may function as a tumor suppressor by inhibiting cell proliferation and invasion in glioma.

Hsa-miR-495 exerts its effects via negative regulation of MYB in glioma. To explore the downstream targets of hsa-miR-495, bioinformatic analysis was performed to identify potential targets. MYB was one of the putative genes identified by bioinformatics prediction, its mRNA 3'-UTR contains a complementary site for the seed region of hsa-miR-495 (Fig. 4A). Furthermore, MYB has been identified as an oncogene, which is associated with progression and development of various types of cancer (11-14). 
$\mathbf{A}$

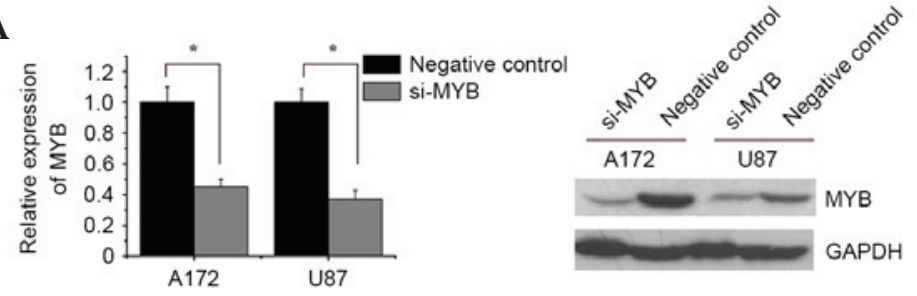

B
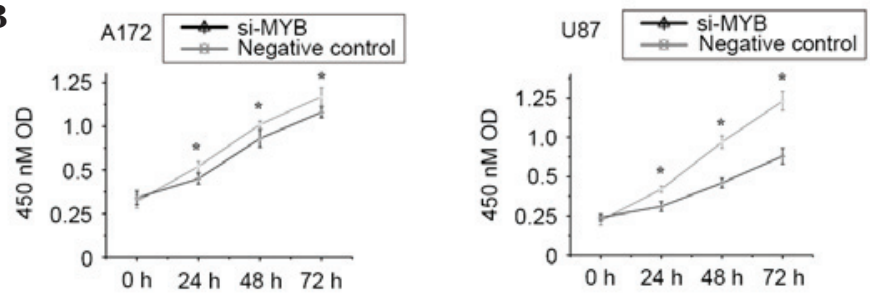

C
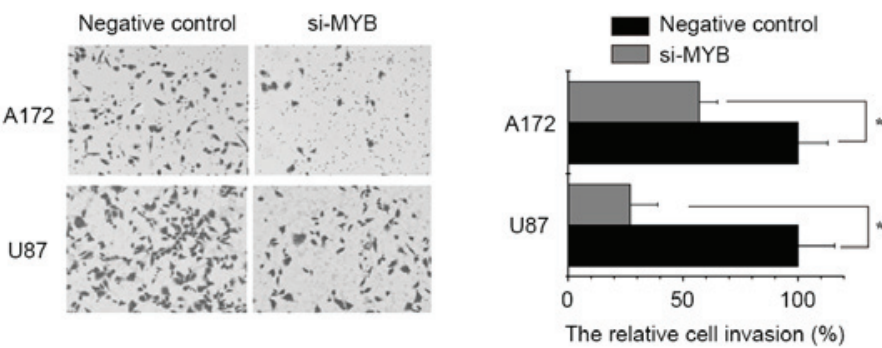

Figure 5. Knockdown of MYB inhibits cell proliferation and invasion in glioma. (A) Quantitative polymerase chain reaction and western blotting were performed to determine the expression levels of MYB in A172 and U87 cells transfected with si-MYB or negative control siRNA. (B) Si-MYB or negative control siRNA-transfected A172 and U87 cells were subjected to CCK-8 assays. * $<0.05$. (C) Si-MYB or negative control siRNA-transfected A172 and U87 cells were subjected to a Transwell invasion assay (magnification, $\mathrm{x} 200)$. ${ }^{*} \mathrm{P}<0.005$. Data are presented as the mean \pm standard deviation $(\mathrm{n}=3$ ). Each experiment was repeated three times. miR, microRNA; si, small interfering RNA; OD, optical density; MYB, v-myb avian myeloblastosis viral oncogene homolog; GAPDH, glyceraldehyde 3-phosphate dehydrogenase.

In order to determine whether MYB was directly regulated by hsa-miR-495, the 3'-UTR fragment of MYB,containing aputative or mutant binding site, was cloned into psiCHECK-2 to construct recombinant plasmids (psiCHECK-2-HMGB1-WT-3'-UTR and psiCHECK-2-HMGB1-MUT-3'-UTR). Subsequently, a luciferase reporter assay was performed on A172 and U87 cells. As presented in Fig. 4B, relative luciferase activity in the psiCHECK-2-HMGB1-WT-3'-UTR group was significantly decreased post-transfection of A172 and U87 cells with hsa-miR-495 mimics $(\mathrm{P}<0.05)$, whereas no notable reduction was detected in the psiCHECK-2-HMGB1-MUT-3'-UTR group. qPCR and western blot analysis were performed to examine the inhibitory effects of hsa-miR-495 on endogenous MYB expression in glioma cells. As shown in Fig. 4C, increased hsa-miR-495 expression markedly inhibited the expression of MYB in A172 and U87 cells. These data suggest that hsa-miR-495 can inhibit MYB expression by directly targeting its mRNA 3'-UTR in glioma.

Knockdown of MYB inhibits cell proliferation and invasion in glioma. To confirm the functional roles of MYB in glioma cells, MYB expression was knocked down in A172 and U87 cells by MYB-specific siRNA (5'-CAGAUGACUGGAAAGUUAUUGCCAA-3' was selected as the optimum MYB-specific siRNA, thus was used for further experiments). The knockdown was confirmed by qPCR and western blotting (Fig. 5A). CCK-8 and Transwell assays indicated that knockdown of MYB expression in A172 and U87 cells was able to significantly inhibit cell proliferation and invasion compared with the control group (Fig. 5B and C), which was similar to the effects of hsa-miR-495 overexpression. These results strongly indicate that hsa-miR-495 acts as a tumor suppressor gene in glioma via the negative regulation of MYB.

\section{Discussion}

Glioma is one of the most devastating types of cancer, since it often exhibits aggressive behavior and cannot be cured by current therapeutic strategies (15). Glioma often develops as a result of genetic alterations that accumulate throughout tumor progression (16). Therefore, elucidation of the molecular mechanisms underlying glioma progression, in particular those associated with cellular proliferation and invasion, is essential to improve understanding regarding the prevention and treatment of glioma (17).

It has previously been reported that miRNAs have an important role in the proliferation and invasion of glioma. For example, hsa-miR-25 inhibits glioma cell proliferation by targeting cyclin-dependent kinase inhibitor 1C (18); hsa-miR-383 regulates proliferation, migration, invasion and apoptosis in human glioma (19); and hsa-miR-320 inhibits cell proliferation by targeting E2F transcription factor 1 in glioma (20). Previous studies have demonstrated that hsa-miR-495 expression is often downregulated in several types of human cancer, including non-small cell lung cancer, 
gastric cancer, and acute myeloid leukemia, thus indicating that hsa-miR-495 may function as a tumor suppressor gene (7-9).

The present study demonstrated that hsa-miR-495 expression was downregulated in glioma tissues and cell lines. Consistent with previous reports, these data suggested that hsa-miR-495 may function as a tumor suppressor gene in glioma by inhibiting cell growth and invasion in A172 and U87 cells. Furthermore, bioinformatics and a luciferase reporter assay revealed that hsa-miR-495 could directly target MYB and negatively regulate its expression.

MYB is a well-acknowledged oncogene in several types of human cancer. Knockdown of MYB contributes to inhibit malignant transformation by regulating genes that participate in numerous aspects of tumorigenesis, including cell growth arrest, invasion inhibition and apoptosis induction (21-24). In the present study, knockdown of MYB expression significantly decreased the proliferation and invasion of A172 and U87 cells, which was similar to the effects of hsa-miR-495 overexpression. These data revealed that hsa-miR-495 suppressed glioma development by targeting MYB, thus providing a potential mechanism underlying post-transcriptional control of glioma.

In conclusion, hsa-miR-495 was downregulated in glioma tissues and cell lines, and acts as a tumor suppressor gene in glioma via the negative regulation of MYB. Therefore, hsa-miR-495 may be considered a potential therapeutic biomarker for the future treatment of glioma.

\section{Acknowledgements}

The present study was supported by the Heilongjiang Province Scientific Research Foundation for Returness (grant no. LC2013C18).

\section{References}

1. Jemal A, Bray F, Center MM, Ferlay J, Ward E and Forman D: Global cancer statistics. CA Cancer J Clin 61: 69-90, 2011.

2. Osborn AG, Salzman KL, Thurnher MM, Rees JH and Castillo M: The new World Health Organization Classification of Central Nervous System Tumors: What can the neuroradiologist really say? AJNR Am J Neuroradiol 33: 795-802, 2012.

3. Furnari FB, Fenton T, Bachoo RM, Mukasa A, Stommel JM, Stegh A, Hahn WC, Ligon KL, Louis DN, Brennan C, et al: Malignant astrocytic glioma: Genetics, biology, and paths to treatment. Genes Dev 21: 2683-2710, 2007.

4. Ambros V: The functions of animal microRNAs. Nature 431: 350-355, 2004.

5. Kim VN: Small RNAs: Classification, biogenesis, and function. Mol Cells 19: 1-15, 2005.
6. Shenouda SK and Alahari SK: MicroRNA function in cancer: Oncogene or a tumor suppressor? Cancer Metastasis Rev 28: 369-378, 2009.

7. Chu H, Chen X, Wang H, Du Y, Wang Y, Zang W, Li P, Li J, Chang J, Zhao G and Zhang G: MiR-495 regulates proliferation and migration in NSCLC by targeting MTA3. Tumour Biol 35: 3487-3494, 2014.

8. Li Z, Cao Y, Jie Z, Liu Y, Li Y, Li J, Zhu G, Liu Z, Tu Y, Peng G, et al: MiR-495 and miR-551a inhibit the migration and invasion of human gastric cancer cells by directly interacting with PRL-3. Cancer Lett 323: 41-47, 2012.

9. Jiang X, Huang H, Li Z, He C, Li Y, Chen P, Gurbuxani S, Arnovitz S, Hong GM, Price C, et al: MiR-495 is a tumor-suppressor microRNA down-regulated in MLL-rearranged leukemia. Proc Natl Acad Sci USA 109: 19397-19402, 2012.

10. Schmittgen TD and Livak KJ: Analyzing real-time PCR data by the comparative C(T) method. Nat Protoc 3: 1101-1108, 2008.

11. Wang W, Wu S, Shi Y, Miao Y, Luo X, Ji M, Yao K and He J: c-MYB regulates cell growth and DNA damage repair through modulating MiR-143. FEBS Lett 589: 555-564, 2015.

12. Liang J, Liu X, Xue H, Qiu B, Wei B and Sun K: MicroRNA-103a inhibits gastric cancer cell proliferation, migration and invasion by targeting c-Myb. Cell Prolif 48: 78-85, 2015.

13. Yang K, He M, Cai Z, Ni C, Deng J, Ta N, Xu J and Zheng J: A decrease in miR-150 regulates the malignancy of pancreatic cancer by targeting c-Myb and MUC4. Pancreas 44: 370-379, 2015.

14. Mets E, Van der Meulen J, Van Peer G, Boice M, Mestdagh P, Van de Walle I, Lammens T, Goossens S, De Moerloose B, Benoit Y, et al: MicroRNA-193b-3p acts as a tumor suppressor by targeting the MYB oncogene in T-cell acute lymphoblastic leukemia. Leukemia 29: 798-806, 2015.

15. Claes A, Idema AJ and Wesseling P: Diffuse glioma growth: A guerilla war. Acta Neuropathol 114: 443-458, 2007.

16. Huse JT and Holland EC: Targeting brain cancer: Advances in the molecular pathology of malignant glioma and medulloblastoma. Nat Rev Cancer 10: 319-331, 2010.

17. Hoelzinger DB, Demuth T and Berens ME: Autocrine factors that sustain glioma invasion and paracrine biology in the brain microenvironment. J Natl Cancer Inst 99: 1583-1593, 2007.

18. Zhang J, Gong X, Tian K, Chen D, Sun J, Wang G and Guo M: MiR-25 promotes glioma cell proliferation by targeting CDKN1C. Biomed Pharmacother 71: 7-14, 2015.

19. Xu D, Ma P, Gao G, Gui Y, Niu X and Jin B: MicroRNA-383 expression regulates proliferation, migration, invasion, and apoptosis in human glioma cells. Tumour Biol 36: 7743-7753, 2015.

20. Sun JY, Xiao WZ, Wang F, Wang YQ, Zhu YH, Wu YF, Miao ZL and Lin YC: MicroRNA-320 inhibits cell proliferation in glioma by targeting E2F1. Mol Med Rep 12: 2355-2359, 2015.

21. Cai W, Li Q, Yang Z, Miao X, Wen Y, Huang S and Ouyang J: Expression of p53 upregulated modulator of apoptosis (PUMA) and C-myb in gallbladder adenocarcinoma and their pathological significance. Clin Transl Oncol 15: 818-824, 2013.

22. Chen RX, Xia YH, Xue TC and Ye SL: Transcription factor $\mathrm{c}-\mathrm{Myb}$ promotes the invasion of hepatocellular carcinoma cells via increasing osteopontin expression. J Exp Clin Cancer Res 29: 172, 2010.

23. Lu H, Wang Y, Huang Y, Shi H, Xue Q, Yang S, He S and Wang H: Expression and prognostic role of c-Myb as a novel cell cycle protein in esophageal squamous cell carcinoma. Clin Transl Oncol 15: 796-801, 2013.

24. Tang R, Li J, Yue M, Liu Z, Feng S, Tang S and Wang T: A correlation analysis of miRNA-34a and its predicted target genes in leukemia. Mol Med Rep 9: 1283-1288, 2014. 